\title{
Pulse Radiolysis Study on Radical Ions of Styrene
}

\author{
Hiroshi YoshidA* and Masato NodA \\ Research Reactor Institute, Kyoto University, Kumatori, Osaka, Japan. \\ Masahiro IRIE
}

Faculty of Engineering, Hokkaido University, Sapporo, Japan.

(Received November 19, 1970)

\begin{abstract}
Pulse radiolysis investigation was carried out on solutions of styrene in 2-methyltetrahydrofuran and $n$-butyl chloride with $1.0-\mu \mathrm{sec}$ electron pulses $(15 \mathrm{Mev})$ at room temperature. Styryl radicals and radical anions formed from the solute were observed from the former solution. In addition to the styryl radicals, the absorption band $\left(\lambda_{\max }=\right.$ $350 \mathrm{~nm}$ ) of cation radicals was observed in the $n$-butyl chloride solution. These radicals are formed by the positive charge transfer from the solvent to the solute styrene, and disappear mainly with the 2 nd order kinetics by recombining with anionic intermediates. However, in the later period, their decay deviates from the 2 nd order kinetics. This results from the superposing 1 st order reaction with rate constant of about $2 \times 10^{6} \mathrm{~mol}^{-1} l \mathrm{sec}^{-1}$, probably due to the addition of styrene to the radical cations. These results are discussed with respect to the initiation process in radiation-induced cationic polymerization of styrene.

KEY WORDS Styrene/Radical Ions/Radiation-Induced Polymerization/ Pulse Radiolysis /
\end{abstract}

The formation of radical anions ${ }^{1}$ and radical cations of styrene was studied by optical absorption $^{1}$ and by electron spin resonance measurements ${ }^{2}$ of 2-methyltetrahydrofuran and butyl chloride irradiated with $\gamma$-rays at $77^{\circ} \mathrm{K}$ in the presence of styrene as solute. Attachment of electrons formed by the ionization of the 2methyltetrahydrofuran glass matrix to the solute molecules resulted in the formation of radical anions, whereas the positive charge transfer from the glass matrix to the solute formed radical cations in the butyl chloride glass.

Short-lived radiolytic products from styrene in liquid phase were observed by the pulse radiolysis technique. ${ }^{3}$ Metz, et al., observed, from pure, dry styrene, rapidly decaying species with absorption maxima, $\lambda_{\max }$, at 370 and $320-$ $330 \mathrm{~nm}$, which they attributed to radical anions and styryl radicals, respectively ${ }^{4}$. Schneider and Swallow examined bulk styrene and styrene solution in cyclohexane and found the absorption due to polymerizing radicals $\left(\lambda_{\max }=320 \mathrm{~nm}\right)$ and that of radical anions $\left(\lambda_{\max }=390 \mathrm{~nm}\right) .^{5}$

* Present address: Faculty of Engineering, Hokkaido University, Sapporo, Japan.
Transient absorptions were observed also in solutions of dioxane, tetrahydrofuran, ${ }^{6}$ tetrachloromethane, aliphatic alcohol, ${ }^{7}$ and water. ${ }^{6}$ However, evidence of cationic species of styrene has not yet been obtained by this technique.

On the other hand, radiation-induced polymerization of styrene has been studied extensively and, when dried rigorously, it proceeds via ionic mechanism. ${ }^{8,9}$ From the study of the effect of added water and other cation scavengers, Ueno, et al., showed that the polymerization can be regarded mainly as a cationic process. ${ }^{8}$ Potter, et al., reached a similar conclusion. ${ }^{9}$

In the present investigation, the pulse radiolysis of styrene solutions was again tried in order to detect cationic species correlated to the cationic polymerization. 2-Methyltetrahydrofuran and $n$-butyl chloride were chosen as solvents to give radical anions and radical cations, because they were proved to be suitable solvents in a frozen glassy state. ${ }^{1,2}$

\section{EXPERIMENTAL}

Pulse Radiolysis Instrument 
For pulse irradiation, an electron linear accelerator (High Voltage Engineering Co., ARCO No. L-1512-G) of the Research Reactor Institute, Kyoto University was used, which provided electron beams of a maximum energy of $25 \mathrm{Mev}$ and a duration of $10 \mathrm{~ns}-4 \mu \mathrm{s}$. The samples were irradiated usually by a $1.0 \mu \mathrm{s}$ of about $15 \mathrm{Mev}$ electrons. Peak current was measured by collecting electrons with a brass block, being $200-300 \mathrm{~mA}$, of which pulse-topulse fluctuation was within $\pm 10 \%$.

An analyzing light beam from a high-pressure xenon lamp (500 watts) was passed through a quartz cell $(20 \phi \times 20 \mathrm{~mm})$, and then to a Bausch and Lomb-type monochrometer (Simadzu Co., 33-86-07) and a photomultiplier (EMI-9558 QB). A wave length region of $300-700 \mathrm{~nm}$ was measurable with a noise level corresponding to $0.2-\%$ light absorption.

\section{Sample Preparation}

$n$-Butyl chloride and 2-methyltetrahydrofuran were distilled several times and dried over $\mathrm{CaH}_{2}$. The former was then dried over baked $\mathrm{BaO}$, and the latter with a $\mathrm{Na}-\mathrm{K}$ alloy. Styrene was washed with aqueous solution of $\mathrm{NaOH}$, distilled, and dried over $\mathrm{CaH}_{2}$ and $\mathrm{BaO}$. After distillation, all materials were treated in a vacuum system less than $10^{-4} \mathrm{mmHg}$, being kept dry.

\section{RESULTS}

\section{n-Butyl Chloride Solutions}

Figure 1 shows representative absorption spectra observed from $n$-butyl chloride solution of styrene after an electron pulse. The difference between the spectra $10 \mu \mathrm{s}$ and $60 \mu \mathrm{s}$ after the pulse indicates short-lived absorption bands with the maxima at $340-350 \mathrm{~nm}$ aud $450-460$ $\mathrm{nm}$. Both bands disappear rapidly. However, the latter seems to have a longer life time. In addition, the intensity ratio of these bands differs from sample, depending on the concentration of styrene. These two bands are, therefore, attributed to different species.

A long-lived absorption band extends from $350 \mathrm{~nm}$ to the ultraviolet region. This is the band already reported by Metz, et al. ${ }^{3}$ and Schneider and Swallow, ${ }^{4}$ and is attributed to styryl radicals and some other free radicals

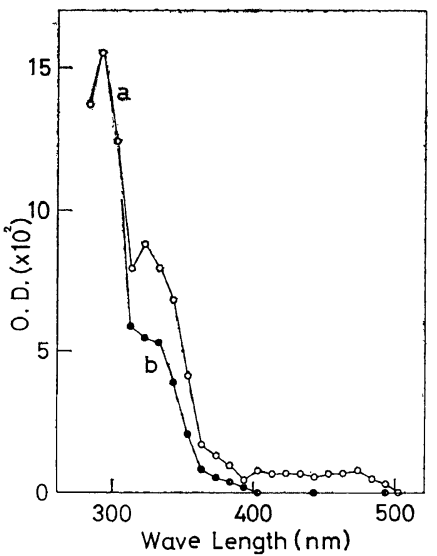

Figure 1. Absorption spectra of $0.3-\mathrm{mol} \%$ solution of styrene in $n$-butyl chloride at $30^{\circ} \mathrm{C}$ : a, 10 $\mu \mathrm{s}$ after $1-\mu \mathrm{s}$ electron pulses; b, $60 \mu \mathrm{s}$ after $1-\mu \mathrm{s}$ electron pulses.

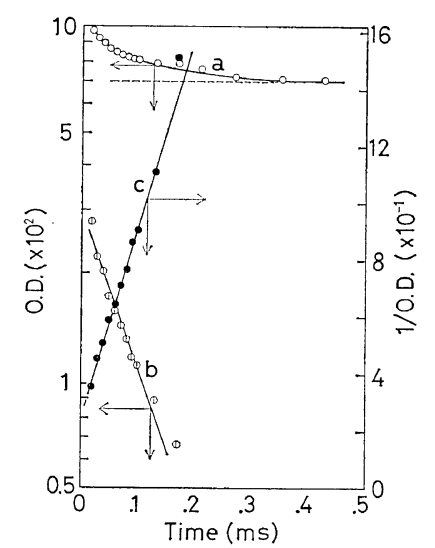

Figure 2. Decay of optical absorption in 0.3-mol\% solution of styrene in $n$-butyl chloride after an $1-\mu \mathrm{s}$ electron pulse measured at $333 \mathrm{~nm}$ and at $30^{\circ} \mathrm{C}$ : a, observed decay composed of fast and slow components; $\mathrm{b}$, the first-order plot; $\mathrm{c}$, the secondorder plot of fast decay components.

formed from styrene. Very stable absorption readily bleached by analizing light ${ }^{9}$ was observed in the present investigation, also.

In order to examine the decay behavior of the short-lived bands, the optical density at 333 $\mathrm{nm}$ was plotted as a function of time after the pulse, as shown in Figure 2 (curve a). The decay curve is divided into two components: the fast component disappears completely within $400 \mu \mathrm{s}$, while the slow component is much 


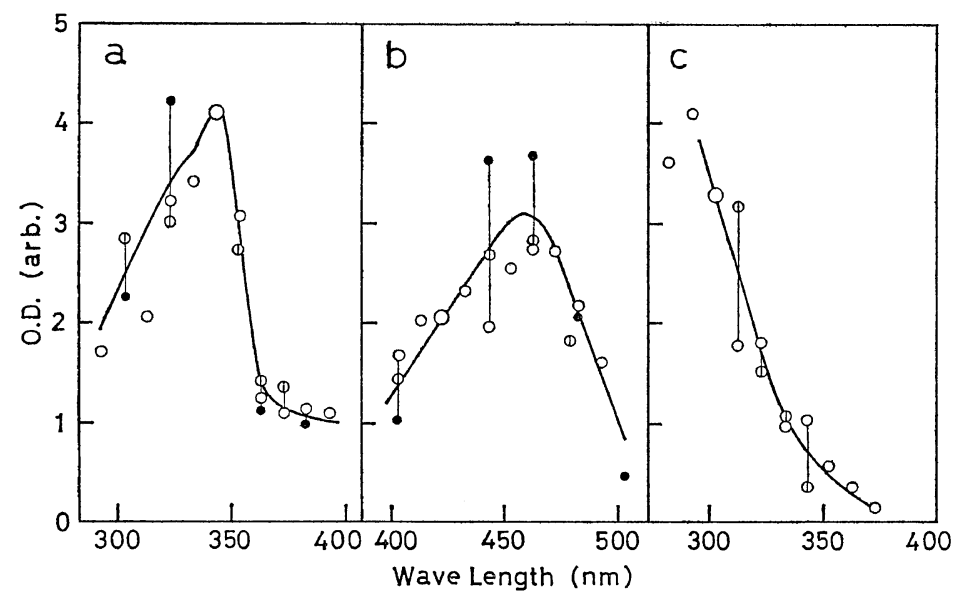

Figure 3. Most probable shape absorption bands observed from styrene solution in $n$-butyl chloride after electron pulses. Band with half-life of (a) a few tens of microseconds, (b) about the same but a little longer than (a), and (c) about $30 \mathrm{~ms}$. Measurements for different concentrations, ( 0.1 , (O) 0.3 and (1) $0.5 \mathrm{~mol} \%$, are normalized at wave lengths indicated by large white circles.

steadier. The 1st order and 2nd order plots of the fast component are shown by curve $b$ and curve c, respectively. Observed results fit better to the 2nd order decay kinetics, though the decay rate becomes higher in the later period.

The analysis of decay curves enables the separation of the bands superposing on one other. The most probable shape of three bands, the short-lived bands with $\lambda_{\max }=350 \mathrm{~nm}$ and $\lambda_{\max }=460 \mathrm{~nm}$ and the long-lived band, is estimated from measurements of three samples, and shown in Figure 3. The wave length at the maximum absorption, $\lambda_{\max }$, is well determined

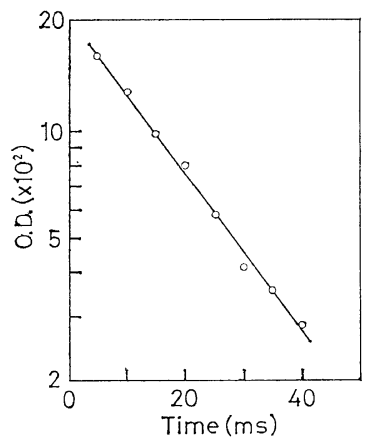

Figure 4. The first-order plot of slow-decay component of optical absorption in $0.3-\mathrm{mol} \%$ solution of styrene in $n$-butyl chloride, determined at $333 \mathrm{~nm}$ and at $30^{\circ} \mathrm{C}$. for the short-lived bands, but not for the longlived band.

The decay of the long-lived band is scarcely dependent on the concentration of styrene, being exponential with a half life of about $15 \mathrm{~ms}$, as shown in Figure 4. The reaction responsible for this decay is ambiguous.

\section{2-Methyltetrahydrofuran Solutions}

2-Methyltetrahydrofuran solutions of styrene give absorption spectra different from those of

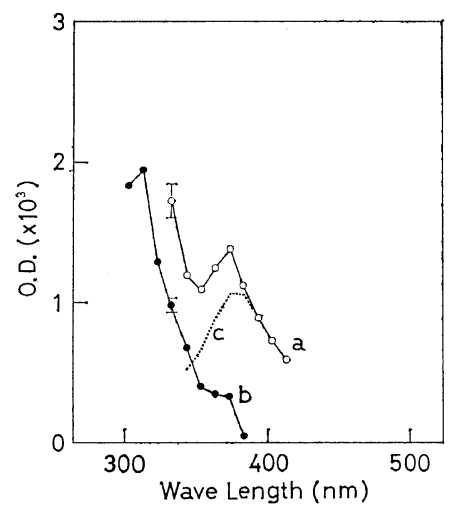

Figure 5. Absorption spectra of 0.4-mol\% styrene solution in 2-methyltetrahydrofuran at $30^{\circ} \mathrm{C}$ : a, $3-\mu \mathrm{s}$ after $1-\mu \mathrm{s}$ electron pulses; b, $8-\mu \mathrm{s}$ after $1-\mu \mathrm{s}$ electron pulses. Difference between $a$ and $b$ is indicated by $c$. 
$n$-butyl chloride solutions, as shown in Figure 5. The spectra are composed of a very short-lived band with a half life of a few micro seconds and the maximum at about $390 \mathrm{~nm}$, and a longlived band extending from $350 \mathrm{~nm}$ to the UV region. The latter is due to both free radicals formed from styrene and some species formed from solvent molecules. The short-lived band agrees well with the $390-\mathrm{nm}$ band observed by Schneider and Swallow ${ }^{5}$ and is compared with the 370-nm band observed by Metz, et al., ${ }^{4}$ from bulk styrene, being attributed to radical anions formed by electron capture of styrene.

\section{DISCUSSION}

\section{Identification of Band due to Radical Cations of Styrene}

In addition to the absorption bands due to styryl radicals and radical anions which were reported, ${ }^{4.5}$ a new band with $\lambda_{\max }$ of $350 \mathrm{~nm}$ was obtained from $n$-butyl chloride solutions in the present investigation. The band is unquestionably due to cationic species, because it disappears when a small amount of 2-methyltetrahydrofuran (cation scanvenger) is added to the $n$-butylchloride solutions. It is reasonably attributed to radical cations formed by positive charge transfer from solvent to solute styrene, because such a cationic process is known from optical obsorption and ESR investigations ${ }^{1.2}$ to dominate in the $n$ - and $s$-butyl chloride matrices at low temperature $77^{\circ} \mathrm{K}$. The shape, as well as $\lambda_{\max }$, of the band is very similar to those of styrene cation radicals formed in an irradiated glassy matrix. ${ }^{1}$

Although another band of the cation radicals should have been observed at about $600 \mathrm{~nm}$ from the glassy matrix, ${ }^{1}$ it was not observable in the present pulse radiolysis study because of the lesser sensitivity of the instrument in the region of longer wave lengths.

The short-lived band with $\lambda_{\max }=460 \mathrm{~nm}$ is always observed when the radical cations are present. The short life suggests that it is due to charged species formed from the radical cations, probably dimer cations. ${ }^{10}$ However, the nature of the species responsible for the band is not elucidated in detail in the present investigation.

\section{Decay of Radical Cations of Styrene}

As the positive charge transfer is a very efficient process in $n$-buthylchloride solvent, principal cationic intermediates are radical cations of styrene. The decay of radical cations may be described by the following equation

$$
-\frac{\mathrm{d}\left[\mathrm{St}^{+}\right]}{\mathrm{d} t}=k_{1}\left[\mathrm{St}^{+}\right]^{2}+k_{2}\left[\mathrm{St}^{+}\right][\mathrm{St}]
$$

The first term of the right-hand side represents the neutralization process between the radical cations and anionic intermediates such as $\mathrm{Cl}^{-}$, and the second term the addition process of styrene to the radical cations. In the pulse radiolysis study, the initial concentration of the radical cations, $\left[\mathrm{St}^{+}\right]$, is rather high, and the neutralization process dominates, showing the 2nd-order decay of radical cations.

Combined with the result of ESR study that the $G$ value of the radical cations of styrene is about 1 in $n$-butyl chloride glass ${ }^{2}$, and with the observed spectrum of the radical cations at $77^{\circ} \mathrm{K}$ (Figure 6 in ref 1), the extinction coefficient is estimated to be about $1.6 \times 10^{3} \mathrm{~mol}^{-1} 1 \mathrm{~cm}^{-1}$ at $333 \mathrm{~nm}$. This leads to the conclusion that the initial concentration of radical cations is about $3 \times 10^{-5} \mathrm{~mol} l^{-1}$ in the experiment shown in Figure 2. If we take the value of $10^{10} \mathrm{~mol}^{-1} l$ $\mathrm{sec}^{-1}$ for $k_{1}$ (diffusion-controlled neutralization process), the second-order decay of the radical cations implies that $k_{2}<10^{7} \mathrm{~mol}^{-1} l \mathrm{sec}^{-1}$.

\section{Initiation Process of Radiation-Induced Polymeri- zation}

The deviation from the 2nd-order kinetics in the later period of decay of the radical cations is thought to be due to the second term of the right-hand side of eq 1 . When $\left[\mathrm{St}^{+}\right]$lowers, the 1st-order process becomes more and more applicable. At one fifth of the initial $\left[\mathrm{St}^{+}\right], k_{1}$ $\left[\mathrm{St}^{+}\right]^{2} \approx k_{2}\left[\mathrm{St}^{+}\right]$[St], though it is a rough estimation (see Figure 2, curve c). This results in $k_{2} \approx 2 \times 10^{6} \mathrm{~mol}^{-1} l \mathrm{sec}^{-1}$.

The propagation rate constant was estimated to be about $10^{7} \mathrm{~mol}^{-1} l \mathrm{sec}^{-1}$ for the radiationinduced cationic polymerization of styrene. ${ }^{11}$ The value denotes the rate of addition of styrene monomer to carbonium ions of chain ends. $k_{2}$ describes the rate of addition of styrene to the radical cation probably to form carbonium ions: 


$$
\begin{aligned}
\left(\mathrm{CH}_{2}=\mathrm{CHC}_{6} \mathrm{H}_{5}\right)^{+} \cdot+\mathrm{CH}_{2}=\mathrm{CHC}_{6} \mathrm{H}_{5} & \\
\rightarrow & \rightarrow \mathrm{CHC}_{6} \mathrm{H}_{5}-\mathrm{CH}_{2}-\mathrm{CH}_{2}-\stackrel{+}{\mathrm{C}} \mathrm{HC}_{6} \mathrm{H}_{5}
\end{aligned}
$$

This is the final step of the initiation process. Because of the resonance state of radical cations, they are less reactive to styrene than carbonium ions and, therefore $k_{2}$ is less than $10^{7} \mathrm{~mol}^{-1} l \mathrm{sec}^{-1}$ (propagation rate constant).

The propagation rate constant was estimated for the bulk polymerization, while $k_{2}$ is obtained in $n$-butyl chloride. The smaller value of $k_{2}$ may be attributed partly to solvation of the cation radicals.

The radical cations are necessarily cationic intermediates primarily formed in bulk styrene irradiated by ionizing radiations. Reaction 2 is the second step of the initiation process, preceded by the ionization step, in radiation-induced cationic polymerization. The observation of cation radicals in the present investigation is closely correlated to the conclusion drawn from the polymerization kinetics data that the radiation-induced polymerization of dried styrene proceeds mainly in cationic mechanism. ${ }^{8,9}$

Acknowledgement. The authors wish to express their sincere thanks to Prof. S. Okamura and Prof. T. Higashimura of the Research Reactor Institute, Kyoto University and Prof. $\mathbf{K}$. Hayashi of Hokkaido University for their valuable suggestions and continuous encouragement, and to Mr. K. Nishimoto for his assistance in operating the linear accerator.

\section{REFERENCES}

1. T. Shida and W. H. Hamill, J. Chem. Phys., 44, 4372 (1966).

2. T. Iwamoto, K. Hayashi, S. Okamura, Ka. Hayashi, and H. Yoshida, Int. J. Radiat. Phys. Chem., 1, 1 (1969).

3. A. J. Swallow, "Radiation Chemistry II," Adv. Chem. Series 82, R. F. Gould Ed., American Chemical Society, Washington, D. C., 1968, p 499.

4. D. J. Metz, R. C. Potter, and J. K. Thomas, J. Polym. Sci., Part A-1, 5, 877 (1969).

5. C. Schneider and A. J. Swallow, Macromol. Chem., 114, 114 (1968).

6. C. Schneider and A. J. Swallow, Macromol. Chem., 114, 172 (1968).

7. K. W. Chambers, E. Collinson, F. S. Dainton, W. A. Seddon, and F. Wilkinson, Trans. Faraday Soc., 1699 (1969).

8. K. Ueno, K. Hayashi, and S. Okamura, Polymer, 7, 431 (1966).

9. R. C. Potter, R. H. Retton, and D. J. Metz, J. Polym. Sci., Part A-1, 5, 877 (1967).

10. B. Badger and B. Brocklehurst, Trans. Faraday Soc., 65, 2582 (1969).

11. Ff. Williams, Ka. Hayashi, K. Ueno, K. Hayashi, and S. Okamura, Trans. Faraday Soc., 63, 1501 (1967). 